\title{
Enzymatic Synthesis of Sucrose-6-acetate by a Novel Immobilized Fructosyltransferase From Aspergillus sp. GX-0010
}

\author{
Qunliang Li ${ }^{1 *}$, Xin Zhang ${ }^{1}$, Xiaobo Guo ${ }^{1}$, Pingjia Yao ${ }^{2}$ and Yuanan Wei ${ }^{2}$ \\ ${ }^{1}$ School of Chemistry and Chemical Engineering, Guangxi University, Nanning, China \\ ${ }^{2}$ The State Key Laboratory of Subtropical Bio-resource Conservation and Utilization, Guangxi University, Nanning, China \\ "Corresponding author: Qunliang Li, School of Chemistry and Chemical Engineering, Guangxi University, Nanning, China. Tel: +86-07713233718, \\ Fax:+86-07713233718, E-mail: liqunliang231@163.com or liqunliang231@gxu.edu.cn
}

Received: 26 Oct. 2015; $\quad$ Revised: 25 Nov. 2017; $\quad$ Accepted: 5 Dec. 2017 Published online: 15 May 2018

\begin{abstract}
Background: Sucralose is an ideal food sweetener and sucrose-6-acetate (s-6-a) is a key intermediate for synthesis of sucralose. Synthesis of s-6-a was studied by free fructosyltransferase (FTase) from Aspergillus oryzae. Because of the limitations of free enzyme in stability and reusability, a FTase obtained from the new isolated Aspergillus sp. GX-0010 was immobilized and investigated for the potential of s-6-a synthesis.

Objectives: The synthesis of s-6-a with sucrose and glucose-6-acetate (g-6-a) by immobilized fructosyltransferase (IFTase) from a novel Aspergillus sp. GX-0010 was studied, and its synthesis conditions were also optimized.

Materials and Methods: Aspergillus sp. GX-0010 was isolated. The effects of reaction time, ratio of g-6-a to sucrose, $\mathrm{pH}$, substrate (sucrose and g-6-a) concentrations, IFTase concentration and temperature on the synthesis of s-6-a were investigated.

Results: IFTase was able to catalyze sucrose and g-6-a to synthesize the s-6-a. Thermal and pH stability of IFTase were promoted once compared to the FTase. The optimal condition for IFTase catalysis was obtained at $50{ }^{\circ} \mathrm{C}, 60$ min reaction time, $\mathrm{pH}$ 6.5, 1:2 ratio of g-6-a to sucrose and 35.0 g.L-1 concentration of enzyme. Under this optimal condition, a g-6-a conversion rate of $24.96 \%$ was reached.

Conclusions: This study showed IFTase has a great potential in the biosynthesis of s-6-a, a key intermediate of sucralose synthesis.

Keywords: Aspergillus sp. GX-0010, Immobilized fructosyltransferase, Sucralose, Sucrose-6-acetate
\end{abstract}

\section{Background}

Sucralose is an ideal food sweetener, which can be widely used to replace sucrose due to its pure sweetness and good heat stability. The preparation of sucrose-6-acetate (s-6-a) is a key step in synthesis of sucralose (1). Currently, the synthesis methods of s-6-a are mainly composed of chemical and enzymatic procedures. S-6-a can be produced with traditional chemical reaction of sucrose in pyridine with acetic anhydride at variable temperatures. This reaction also produces kinds of impurities, including monoacylates, some higher acylates, and toxic substances, which requires complicated separation techniques for the pure s-6-a (2). Enzymes, mainly fructosyltransferase (FTase) and lipase, have been used in sucralose synthesis. Cross-linked enzyme aggregates and immobilized lipase have been used in the biosynthesis of s-6-a (34). Additionally, use of FTase in transferring a fructosyl residue on either sucrose or glucose-6-acetate (g-6-a) to produce s-6-a have been reported (5-8). The FTase that has been used to date is either under patent restrictions, e.g. Bacillus megaterium NCIB 11871 and Aureobasidium pullulans, or is limited for the reaction in time-cost (120 h) that makes the process non-economical $(5,9)$. The FTase from Aspergillus oryzae was recently purified and characterized, suggesting it having the potential to be a biocatalyst for the synthesis of s-6-a (10). In addition, a FTase was also reported for catalyzing sucrose and glucose-6-acetate to synthesize s-6-a (11), but its practical application is limited due to the poor stability and nonreusability.

Copyright (C 2017 The Author(s); Published by National Institute of Genetic Engineering and Biotechnology. This is an open access article, distributed under the terms of the Creative Commons Attribution-NonCommercial 4.0 International License (http://creativecommons.org/licenses/ by-nc/4.0/) which permits others to copy and redistribute material just in noncommercial usages, provided the original work is properly cited. 


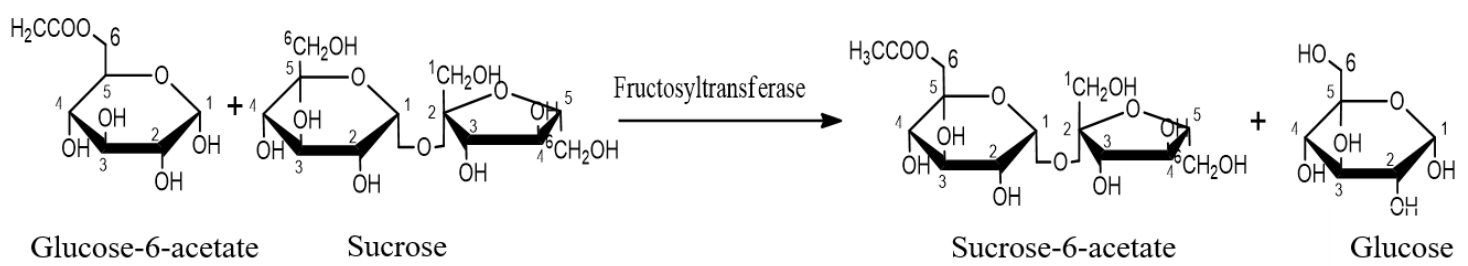

Figure 1. The synthesis of s-6-a catalyzed by FTase

\section{Objectives}

Aspergillus sp. GX-0010 was obtained in our laboratory. In preliminary experiments, FTase from Aspergillus sp. GX0010 was able to catalyze sucrose and g-6-a to synthesize s-6-a, a key intermediate for producing sucralose, as shown in Figure 1. Considering the advantages of immobilized enzymes in enzyme activity, storage stability, and efficient separation over the free enzymes (12-13), an immobilized fructosyltransferase (IFTase) from Aspergillus sp. GX0010 was used as a catalyst to synthesize s-6-a and the synthesis conditions were also optimized.

\section{Materials and Methods}

\subsection{Reagents}

The standard samples of fructose and sucrose were from Sigma Co. Ltd. S-6-a was purchased from Toronto Research Chemicals Inc., Canada. The g-6-a was prepared in our laboratory.

\subsection{Microbial Strain and Cultivation}

Aspergillus sp. GX-0010 obtained by mutation in our laboratory was inoculated into $250 \mathrm{~mL}$ culture medium containing sterile sucrose 24.0 g.L. $\mathrm{L}^{-1}$, yeast extract 5.0 g. $\mathrm{L}^{-1}$, and $\mathrm{K}_{2} \mathrm{SO}_{4} 1.0$ g. $\mathrm{L}^{-1}$ (pH 6.5) in a $500 \mathrm{~mL}$ flask and incubated at $28^{\circ} \mathrm{C}$ for $32 \mathrm{~h}$ with shaking speed of 150 rpm.

\subsection{Preparation of Immobilized Enzyme with Chitosan Gel in Glutaraldehyde}

The mycelium was collected and washed by phosphate buffer ( $\mathrm{pH}$ 6.5), then disrupted by bead mill. The cell debris was removed by centrifugation at $4{ }^{\circ} \mathrm{C}, 8944 \times \mathrm{g}$ for $60 \mathrm{~min}$. Crude enzyme obtained from supernatant was primarily purified by Ultrafilter at $4{ }^{\circ} \mathrm{C}$. The crude FTase was immobilized according to (14). Briefly, $1.0 \mathrm{~g}$ chitosan was dissolved in $2 \%(\mathrm{~m} / \mathrm{v})$ acetic acid for $12 \mathrm{~h}$, then filtered with filter paper. The filtrate was diluted by 4 times in volume, and neutralized with $2.0 \mathrm{moL} . \mathrm{L}^{-1} \mathrm{NaOH}$. Chitosan-gel was precipitated via vigorous agitation and washed to neutrality with distilled water, then dehydrated via suction filtration. Chitosan-gel was mixed with crude FTase, and the mixture was added to a flask containing $20 \mathrm{~mL}$ glutaraldehyde solution $(0.2 \% \mathrm{w} / \mathrm{v})$ and shaken at $40 \mathrm{rpm}$ for $8 \mathrm{~h}$ at $25^{\circ} \mathrm{C}$. After cross-linking reaction, the prepared of IFTase was washed with distilled water to remove excess glutaraldehyde, then stored in a 0.1 moL.L.- sodium acetate buffer ( $\mathrm{pH} 5.5)$ at $4{ }^{\circ} \mathrm{C}$.

\subsection{Catalytic Conditions of IFTase}

Catalytic conditions including the molar ratio of g-6-a to sucrose, substrate concentrations, reaction time, $\mathrm{pH}$, and temperature were optimized. All reaction volume was in $50 \mathrm{~mL}$ phosphate buffer. To investigate the reusability potential, IFTase was separated from the products by filtration and washed two times with 0.1 moL. $\mathrm{L}^{-1}$ sodium acetate buffer ( $\mathrm{pH}$ 5.5) and dried under vacuum.

\subsection{Definition of the Activity Unit of the Immobilized Enzyme}

One unit ( $1 \mathrm{U})$ of the immobilized enzyme activity was defined as the amount of enzyme that required for catalyzing $1 \mu \mathrm{moL}$ g-6-a per minute. The activity of IFTase was the unit of $\mu \mathrm{moL}$ of s-6-a/min via per IFTase (g), abbreviated as $\mathrm{U} \cdot \mathrm{g}^{-1}$.

\subsection{Analyzing Methods}

The catalyzing reaction of IFTase for sucrose was measured by HPLC (Waters 600 HPLC pump, Waters 717 plus Autosampler, Alltech 2000 ELSD Detector).The analysis column was Hypersil $\mathrm{NH}_{2}(4.6 \times 300 \mathrm{~mm})$. Mobile phase was the mixture of acetonitrile: water $(75: 25)$. Flow rate was $1.0 \mathrm{~mL} \cdot \mathrm{min}^{-1}$ in total of $10 \mu \mathrm{L}$. Column temperature was set to $30^{\circ} \mathrm{C}$ and run time for the elution was $15 \mathrm{~min}$.

The contents of different components were determined by the normalization method according to the HPLC peak. The theoretical and measured contents of s-6-a were detected according to the contents of g-6-a. The molar conversion rate of g-6-a was calculated based on the following formula (11).

$$
\begin{aligned}
& g-6-\text { a molar conversion rate }=\frac{n(s-6-a) \text { measure values }}{n(s-6-a) \text { theoretical values }} \\
& =\frac{w(g-6-a) / M(g-6-a)}{w(g-6-a) / M(g-6-a)+w(g-6-a) / M(g-6-a)} \times 100
\end{aligned}
$$

In formula, $n$ : number of moles, $w$ : mass number, $M$ : molar mass. 


\subsection{Statistical Analysis}

Data, except for HPLC, was the mean value of three replicates. The coefficient of variation was controlled until it did not exceed 5\%. The graphs were drawn via Origin 8.0.

\section{Results}

\subsection{The Identification of Standard Samples and Catalyzing Products}

The retention time of the standard samples of g-6-a, sucrose and s-6-a was detected by HPLC (Fig. 2A). Linear equation in terms of the correlation between concentration of standard s-6-a and its peak area was conducted. The peak areas of serial dilutions of the standard s-6-a $(1.0,2.0$, 3.0, 4.0 and $5.0 \mathrm{mg} \cdot \mathrm{mL}^{-1}$ ) were 238836, 593824, 867463, 1199921 and $1527864 \mathrm{mV} \cdot \mathrm{S}$ respectively determined by HPLC. The linear equation was $y=318415 x-69664$, $\left(R^{2}\right.$ $=0.9988$ ).

After enzyme inactivated in boiling water for $10 \mathrm{~min}$, cooled, centrifuged and diluted, the reaction samples were analyzed by HPLC in the same chromatographic conditions as standard samples did. The g-6-a, s-6-a and sucrose were identified in the catalyzing solution by FTase based on the retention time in Figure 2B in which the g-6-a was the remnant reaction substrate and the sucrose derived from the culture medium and the reaction substrate. The peak at retention time of $6.087 \mathrm{~min}$ indicated that s-6-a was successfully synthesized and the yield of s-6-a can be calculated according to its peak area.
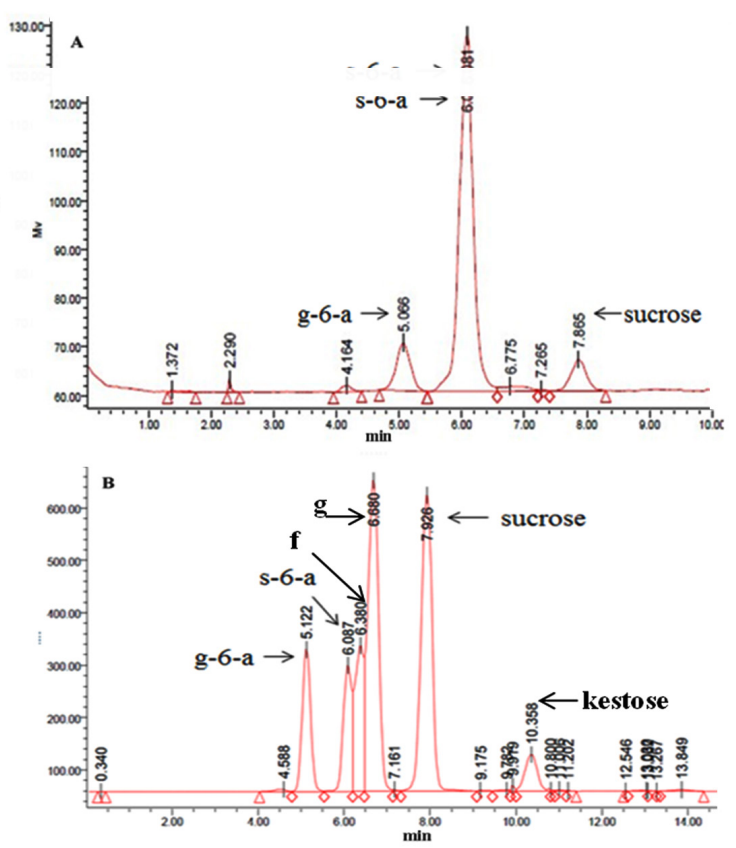

Figure 2. The HPLC spectrum of standard samples (A) and catalyzing products (B). g-6-a: glucose-6-acetate, s-6-a: sucrose-6-acetate, g: glucose, f: fructose.

\subsection{Effect of Temperature and $p H$ on FTase and IFTase}

The effect of temperature on the FTase and IFTase activities was investigated by incubating enzymes at various temperatures $\left(30-60{ }^{\circ} \mathrm{C}\right)$ in buffer solution $(\mathrm{pH}$ 5.5). The appropriate temperature for IFTase was between
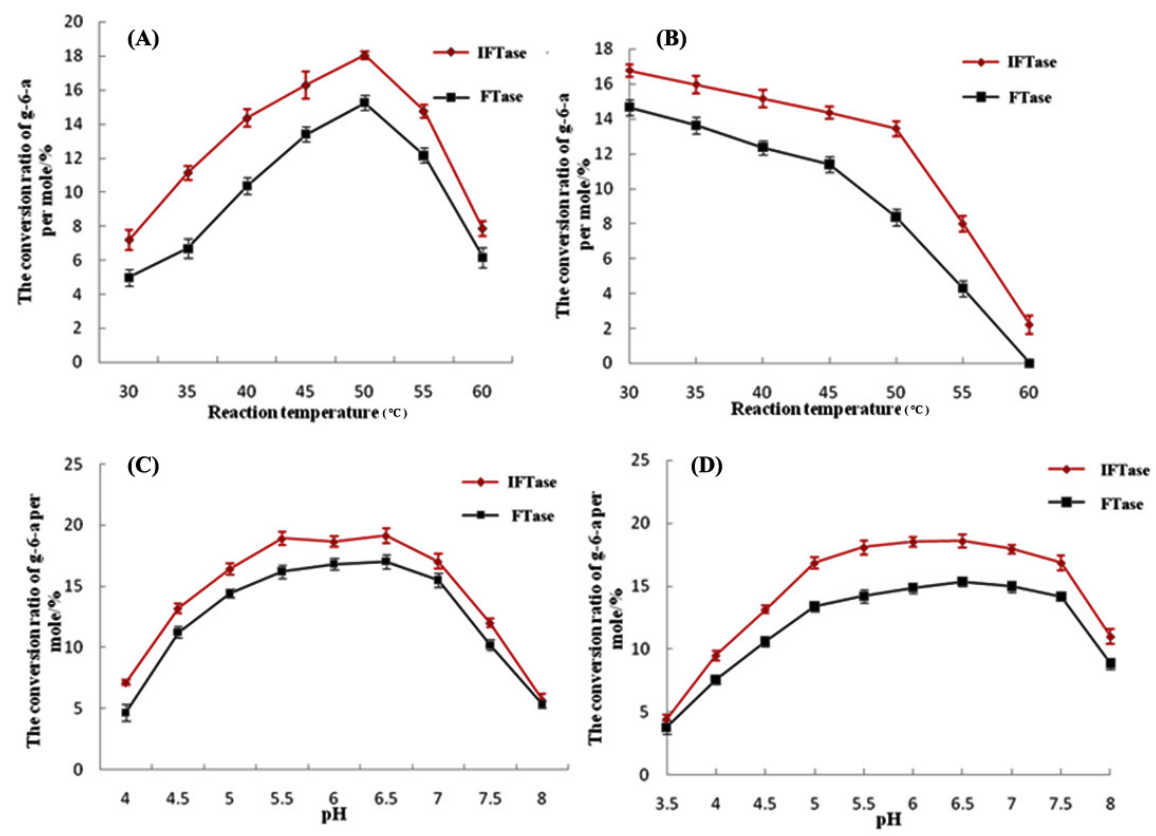

Figure 3. Effect of temperature and $\mathrm{pH}$ on the enzymes' activities and stabilities. A, the effect of temperature on enzymes' activities; B, the effect of temperature on enzymes' stabilities; C, the effect of $\mathrm{pH}$ on enzymes' activities; $\mathrm{D}$, the effect of $\mathrm{pH}$ on enzymes' stabilities. 

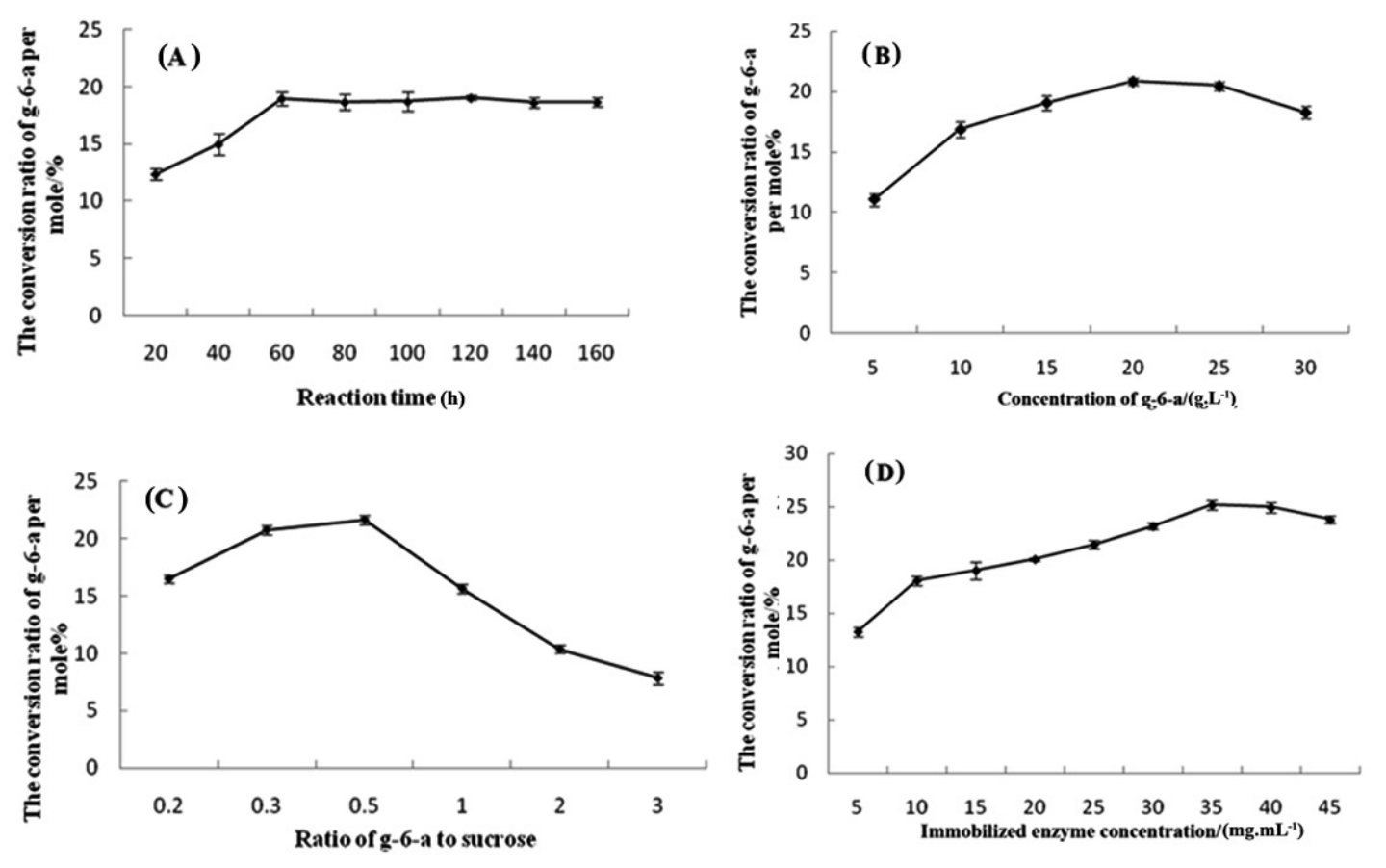

Figure 4. The optimization for IFTase catalyzing reaction. A, optimization of reaction time; B, optimization of g-6-a concentration; C, optimization of reactants ratio; D, optimization of IFTase concentration.

40-50 ${ }^{\circ} \mathrm{C}$ and the optimum temperature was at $50{ }^{\circ} \mathrm{C}$ (Fig. 3A). FTase had the higher activity between 45-50 ${ }^{\circ} \mathrm{C}$. An extended appropriate temperature range of IFTase was observed compared to FTase. The thermal stability of FTase and IFTase was assessed by incubating the enzymes at various temperatures $\left(30-60{ }^{\circ} \mathrm{C}\right)$ in buffer solution at $\mathrm{pH}$ 5.5 for $60 \mathrm{~min}$ (Fig. 3B). The conversion of g-6-a in IFTase was higher than FTase during the different temperature range, indicating that thermal stability of IFTase was promoted relative to FTase.

The effect of $\mathrm{pH}$ on the FTase and IFTase activity was studied by incubating the enzymes in $\mathrm{pH}$ range of 4-8 at $50{ }^{\circ} \mathrm{C}$ (Fig. $3 \mathrm{C}$ ). The appropriate $\mathrm{pH}$ range for both FTase and IFTase was 5.0-7.0 and the optimal $\mathrm{pH}$ was 6.5 . The enzymes were incubated at $50{ }^{\circ} \mathrm{C}$ in buffer solution at $\mathrm{pH}$ 3.5-8.0 for $60 \mathrm{~min}$ to assess the $\mathrm{pH}$ stability of FTase and IFTase activity which were stable at $\mathrm{pH}$ 5.0-7.5, then declined when $\mathrm{pH}$ was above 7.5 (Fig. 3D). The $\mathrm{pH}$ stability of IFTase was enhanced after immobilization.

\subsection{Effects of Catalytic Conditions on g-6-a Conversion Rate Via IFTase}

The conversion rate of g-6-a to s-6-a was used as an index to optimize catalytic time, concentration of g-6-a, ratio of g-6-a to sucrose and concentration of IFTase (Fig. 4).
The effect of catalytic time on the conversion rate of g-6-a was investigated in the range from 20 to $160 \mathrm{~min}$ at $50^{\circ} \mathrm{C}$, $\mathrm{pH} \quad 6.5,15$ g.L. ${ }^{-1}$ of g-6-a, 1:3 of g-6-a to sucrose $(\mathrm{m} / \mathrm{m})$ and $20 \mathrm{~g} . \mathrm{L}^{-1}$ of IFTase concentration. Result revealed that conversion rate of g-6-a was enhanced gradually with increasing time from 20 to $60 \mathrm{~min}$, and reached the peak at $60 \mathrm{~min}$ (Fig. 4A). The effect of g-6-a concentration on the conversion rate of g-6-a was investigated in the range from 5 to $30 \mathrm{~g} . \mathrm{L}^{-1}$ at $50^{\circ} \mathrm{C}, \mathrm{pH} \quad 6.5,60 \mathrm{~min}, 1: 3$ of g-6-a to sucrose $(\mathrm{m} / \mathrm{m})$ and 20 g.L. $\mathrm{L}^{-1}$ of IFTase concentration. When the g-6-a concentration increasing from 5 to 20 g.L. ${ }^{-1}$, the conversion rate of g-6-a was promoted gradually, then reached the maximum of $20.8 \%$ (Fig. 4B). The ratio of g-6-a to sucrose affected the conversion of g-6-a significantly certificated by the catalytic reaction at $50{ }^{\circ} \mathrm{C}, \mathrm{pH} 6.5,60$ min, 20 g.L. $\mathrm{L}^{-1}$ of g-6-a and 20 g.L. $\mathrm{L}^{-1}$ of enzyme concentration. At the beginning, the conversion rate of g-6-a increased and then decreased with inclining of the ratio of g-6-a to sucrose. The maximum conversion rate was observed at the ratio of 1:2 (Fig. 4C). The result in Figure 4D revealed that the conversion rate of g-6-a increased gradually, when IFTase concentration was between 5.0 to $45.0 \mathrm{~g} . \mathrm{L}^{-1}$ with the catalytic reaction at $50^{\circ} \mathrm{C}, \mathrm{pH} 6.5,60 \mathrm{~min}, 20.0 \mathrm{~g} . \mathrm{L}^{-1}$ of g-6-a and 1:2 of g-6-a to sucrose. The conversion rate of g-6-a decreased gradually after it above 35.0 g. $\mathrm{L}^{-1}$. 
4.4. Storage Stability and Reusability of IFTase

The IFTase was kept at $4{ }^{\circ} \mathrm{C}$ and maintained $90.1 \%$ of its initial activity after 6 months storage. In the first 30 days, the activity of IFTase decreased slightly suggesting IFTase had a good storage stability (data was not shown). The reusability of IFTase in synthesizing s-6-a was profiled (Fig. 5). A higher of g-6-a conversion ratio at $92.3 \%$ compared to the initial conversion ability was maintained after 4 cycles of IFTase reusing. The conversion of g-6-a decreased by $50.5 \%$ after 15 cycles of IFTase reusing, suggesting the reutilized cycle of IFTase was 15 . The result indicated that IFTase had a good storage stability and reusability, which was a foundation for its large-scale industrial application.

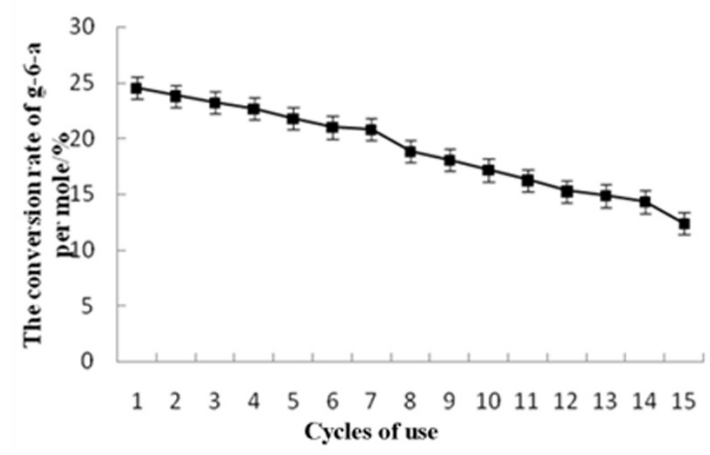

Figure 5. The effects of recyclable use on IFTase activity. All the reaction conditions of the enzyme recyclable use are described in section 3.4 in Materials and Methods. The results showed that the conversion of g-6-a decreased only by $50.5 \%$ after 15 recyclable times of IFTase.

\section{Discussion}

In this study, the activity and optimal conditions of IFTase from Aspergillus sp. GX-0010 were investigated. The results indicated that IFTase could catalyze the sucrose and g-6-a to synthesize the s-6-a. The thermal and $\mathrm{pH}$ stability of IFTase were improved compared to the FTase. The optimal ratio of g-6-a to sucrose of 1:2 was in accordance to the result (1). A higher ratio of g-6-a to sucrose restrained the production of fructose, resulting from the inhibition of substrate to IFTase (Fig. 4C). A steric hindrance from IFTase might has been existed which led to a decrease in the g-6-a conversion rate, when the enzyme concentration above 35.0 g.L. ${ }^{-1}$ (Fig. 4D). The optimal condition was obtained as follows: 50 ${ }^{\circ} \mathrm{C}, 1 \mathrm{~h}$ reaction, $\mathrm{pH} 6.5$, ratio of g-6-a to sucrose of $1: 2$, and enzymatic concentration of 35.0 g. $\mathrm{L}^{-1}$. Under these optimal conditions, the g-6-a conversion rate reached $24.96 \%$. This yield is comparable to the two related reports of s-6-a synthesis using FTase from Aspergillus (10-11). In one report, a novel FTase from Aspergillus oryzae ZZ-
01 was purified and characterized. The purified enzyme led to an overall conversion of $32.0 \%$ rate using g-6-a and sucrose as substrates under the optimal conditions (10). In the other report, Han et al. illustrated the crude FTase from Aspergillus oryzae catalyzed s-6-a synthesis with a g-6-a molar conversion rate of 26.9\% (11). However, bad stability and no-reusability limited the application of free enzyme. The stability and reusability of IF Tase in this work showed that the immobilized enzyme overcame the disadvantages of free enzymes.

Notably, IFTase displayed both conversion and hydrolysis activity in a sucrose reaction system, leading to fructose and glucose formation. The produced fructose can be transferred to sucrose form fructooligosaccharides as well (14-16). Therefore, the sucrose can be a competitive inhibitor of g-6-a in the reaction for synthesizing s-6-a. How to inhibit the kestose production for increasing s-6-a yield will be concerned in the future study.

\section{Acknowledgements}

The authors would like to thank other members of our laboratory for their helpful discussion.

\section{Authors' Contribution}

Qunliang Li, Xin Zhang, Pingjia Yao and Yuanan Wei designed the experiments. Xin Zhang performed the experiments. Qunliang Li, Xin Zhang, and Xiaobo Guo analyzed the data and wrote the manuscript.

\section{Funding/Support}

This research was supported by the grants Natural Science Foundation of Guangxi (2011GXNSFC018008) and the Graduate Innovation Fund of Guangxi (GXU11T32588).

\section{References}

1. Jones JD, Hacking AJ, Cheetham PSJ. Biological method for protection of 6-position of sucrose and its use in synthesis of disaccharide high-intensity sweetener. Biotechnol Bioeng. 1992;39(2):203-210. doi: 10.1002/bit.260390212

2. Sankey GH, Vernon NM, Wingard J. Catalyzed sucrose-6-ester process. US Patent: 5,470,969. November 28, 1995.

3. Yang X, Zheng P, Ni Y, Sun Z. Highly efficient biosynthesis of sucrose-6-acetate with cross-linked aggregates of lipozyme TL 100 L. J Biotechnol. 2012;161(1):27-33. doi:10.1016/j. jbiotec.2012.05.014

4. Zhong X, Qian J, Guo H, Hu Y, Liu M. Biosynthesis of sucrose6-acetate catalyzed by surfactant-coated Candida rugosa lipase immobilized on sol-gel supports. Bioproc Biosyst Enq. 2014;37(5):813-818. doi:10.1007/s00449-013-1053-9

5. Cheetham PS, Dordick JS, Hacking AJ, Khan RA, Mufti KS, Rathbone EB. Tetrachlororaffinose and its use in the preparation of sucralose. US Patent: 4,826,962. May 2, 1989.

6. Van Balken JAM, Van Dooren ThJGM, van den Tweel WJJ, Kamphuis J, Meijer EM. Production of 1-kestose with intact mycelium of Aspergillus phoenicis containing sucrose-1Ffructosyltransferase. Appl Microbiol Biotech. 1991;35(2):216- 


\section{Li Q et al.}

221. doi: 10.1007/BF00184689

7. Maiorano AE, Piccoli RM, da Silva ES, de Andrade Rodrigues MF. Microbial production of fructosyltransferases for synthesis of pre-biotics. Biotechnol Lett. 2008;30(11):1867-1877. doi: 10.1007/s10529-008-9793-3

8. Ritsema T, Verhaar A, Vijn I., Smeekens S. Using natural variation to investigate the function of individual amino acids in the sucrose-binding box of fructan: fructan 6G-fructosyltransferase (6G-FFT) in product formation. Plant Mol Biol. 2005;58(5):597-607. doi: 10.1007/s11103-005-65045

9. Aurora S, Ratnam R, Subramaniyam P. Method of producing sucrose-6-acetate by whole-cell biocatalysis: US Patent: 11,992,235. September 21, 2006.

10. Wei T, Yu X, Wang Y, Zhu Y, Du C, Jia C, Mao D. Purification and evaluation of the enzymatic properties of a novel fructosyltransferase from Aspergillus oryzae: a potential biocatalyst for the synthesis of sucrose 6-acetate. Biotechnol Lett. 2014;36(5):1015-1020. doi: 10.1007/s10529-014-1457-x

11. Han Y, Liu G, Huang D, Qiao B, Chen L, Guan L, Mao D. Study on the synthesis of sucrose-6-acetate catalyzed by fructosyltransferase from Aspergillus oryzae. $N$ Biotechnol.2011;28(1),14-18. doi: 10.1016/j.nbt.2010.07.007

12. Wei Y, Yao P, Xie Q, Tang J. Study on characteristics and thermostability of immobilized fructosyltransferase. J Guangxi Univ. (Nat SciEd ). 2001;27(1):1-4.

13. Wei Y. Yao P, Xie Q, Tang J. The comparative study of enzymatic properties between the free and immobilized fructosyltransferase. Chin J Biochem Mol Biol. 2000;17(4):501505.

14. Wei Y, Xie Q, Yao P, Dong Y, Tang J. Method of producing fructooligosaccharide by immobilized fructosyltransferase. $C N$ Patent: 65624C. 2004.

15. Wei Y, Li M, Hai Y, Jiang S, Li T. Study on the production of fructooligosaccharide by immobilized enzyme. Food Ferment Ind. 1995;4:12-16.

16. Ganaie MA, Rawat HK, Wani OA, Gupta US, Kango N. Immobilization of fructosyltransferase by chitosan and alginate for efficient production of fructooligosaccharide. Process Biochem. 2014;49(5):840-844. doi: 10.1016/j. procbio.2014.01.026 